\title{
COMPORTAMENTO DO ÍON MAGNÉSIO EM PROVA TRIATHLON MEIO-IRONMAN
}

\author{
BEHAVIOR OF THE ION MAGNESIUM \\ IN TRIATHLON HALF-IRONMAN COMPETITION
}

\begin{abstract}
AUTOR
Marcos Bürger-Mendonça'

${ }^{1}$ Graduado em Educação Física pela UNESA
\end{abstract}

COMPORTAMENTO DO ÍON MAGNÉSIO EM PROVA TRIATHLON MEIO-IRONMAN 4(2): 55-60

\section{PALAVRAS-CHAVE}

triathlon meio-ironman; endurance; magnésio; eletrólitos.

\section{KEYWORDS}

triathlon half-ironman; endurance; magnesium; electrolytes.

\section{RESUMO}

0 presente estudo teve como objetivo analisar a resposta do íon magnésio pré e pós-competição de triathlon em atletas do sexo masculino, na distância meio-ironman através da analise bioquímica da concentração do íon magnésio no plasma sangüíneo. Participaram do presente estudo 10 voluntários do sexo masculino fisicamente ativos, divididos em 2 grupos controle e experimental. Foram submetidos a duas coletas de aproximadamente $25 \mathrm{ml}$ de sangue, após jejum de oito horas na posição sentada e imediatamente ao término da prova de cada atleta. Para análise estatística foi utilizado o software SPSS 11.0, para o teste T-student com a mesma amostra, tendo o valor de $\mathrm{P}<0.05$ aceito como significante. Os valores após a competição entre o grupo controle e o grupo experimental apresentou-se uma diferença significativa na concentração plasmática de magnésio $(P=0,005)$. Sendo comparados os valores da concentração plasmática de magnésio no grupo experimental antes e após a competição apresentaram-se também uma diferença significativa $(P=0,005)$, com uma redução média de 35,23\%. Na comparação de valores pós-competição entre o grupo experimental e controle houve uma diferença significativa na concentração plasmática de magnésio, podendo assim inferir que o exercício de longa duração causa uma depledação das reservas de magnésio do organismo.
ABSTRACT

The present study it had as objective to analyze the response of the ion magnesium before and after-competition of triathlon in athlete of the masculine sex, in the half-ironman distance, through it biochemistry analyzes of the concentration of the ion magnesium in the plasma blood. Ten physically active volunteers of the masculine sex participated in the present study, divided in two groups: control and experimental. The integrants of the experimental group was submitted to two blood collections (approximately $25 \mathrm{ml}$ ), after eight hours dietary restriction in the seated position. The second blood sample was collected immediately at ending of the competition of each athlete $(t=1)$. For statistical analysis was used the software SPSS 11.0, and T-student test for statistical comparisons between the two groups ( $P<0.05$ accepted as significant). When compared the values before the competition between the control group and experimental group a significant difference in the concentration of plasma magnesium $(P=0,005)$ was observed. When compared the values of plasma magnesium concentration in the experimental group before and after competition a significant difference $(P=0,005)$, with an average reduction of $35,23 \%$ was observed. In the comparison of values after-competition between the experimental group and control, a significant difference in the plasma magnesium concentration was detected, indicating that the exercises of long duration cause a depletion of magnesium reserves of the organism. 


\section{INTRODUĈ̣̃O}

Os minerais, tanto os traços como os macroelementos, estão envolvidos no funcionamento do corpo, sendo componentes chaves no metabolismo celular desde o nível mais baixo de funcionamento até a atividade funcional dos grandes órgãos (Speich; Pineau; Ballereau, 2001). 0 magnésio $\left(\mathrm{Mg}^{2+}\right)$ e um mineral essencial e co-fator de mais de 300 reações enzimáticas $(B o h l ;$ Volpe, 2002), sendo este um íon metálico divalente com número atômico 12, massa de $24.305 \mathrm{~g} \mathrm{~mol}^{-1}$ e uma densidade de $1,74 \mathrm{~g} \mathrm{~mL}^{-1}$, e o quarto mais abundante cátion no corpo (Wolf; Cittadini, 2003) e é também o segundo cátion intracelular mais abundante após o potássio (Deuster et al., 1987), exercendo vários papéis vitais no metabolismo. A deficiência ou a baixa da concentração plasmática de $\mathbf{M g}^{2+}$ pode afetar a tolerância ao exercício [Zimmermann, 2003] e a função imune (Gay; Meydani, 2001; Speich; Pineau; Ballereau, 2001).

Atualmente, o interesse em relação ao $\mathrm{Mg}^{2+}$ tem aumentado, pois diversos estudos bioquímicos têm sido realizados em competicões desportivas de longa duração, especialmente nos eventos de ultra-endurance (com mais de uma hora de duração] [Warburton et al., 2002). Segundo Peters (2003), exercícios classificados como ultra-endurance apresentam uma duração superior a 4 horas. 0 presente estudo tem como objetivo analisar as diferenças de concentração sanguínea do $\mathrm{Mg}^{2+}$ antes e após uma competição de triathlon em atletas do sexo masculino.

\section{METODOLOGIA}

Participaram do presente estudo dez voluntários do sexo masculino fisicamente ativos. Divididos em dois grupos: controle e experimental. $\mathbf{O}$ grupo experimental foi composto por cinco voluntários do sexo masculino com idade média de $27,20 \pm 6,68$ anos que executaram o experimento que consistia de uma prova de triatlhon meio-ironman, com as seguintes distâncias: $1.9 \mathrm{~km}$ de natação, $90 \mathrm{~km}$ de ciclismo e $21 \mathrm{~km}$ de corrida totalizando uma distância de 113 km. 0 grupo controle foi composto por cinco voluntários do sexo masculino com idade média de 26,40 5 ,22 anos, que estavam presentes no local do experimento sendo que eles não praticaram o triathlon.

Realçamos que os voluntários participantes do experimento neste trabalho não eram fumantes ou consumidores de drogas proibidas pelo Comitê Olímpico Internacional (COI). Esse fato foi verificado através de um questionário respondido previamente pelos voluntários. 0 consumo de alimentos, água e repositores hidroeletróliticos foi realizado de forma ad libitum pelos voluntários.

Todos os voluntários foram informados sobre os procedimentos que seriam realizados e assinaram o termo de livre consentimento e esclarecimento (TLCE) onde eles poderiam deixar o estudo a qualquer momento.

\section{Procedimentos de coleta}

Os Integrantes do experimento foram submetidos a duas coletas de aproximadamente $25 \mathrm{~mL}$ de sangue, após jejum de oito horas, no período entre 6 e 7 horas da manhã na residência do voluntário $(t=0)$ na posição sentada e imediatamente ao término da prova de cada atleta, sendo esta coleta realizada na tenda médica do evento. As amostras de sangue foram coletadas por meio de punção da veia basílica mediana direita, com auxilio de torniquete e com o local previamente esterilizado com álcool $70 \%$, de acordo com as Recomendações da Sociedade Brasileira de Patologia Clínica e Medicina Laboratorial (2005) para coleta de sangue venoso. Foram utilizadas agulhas BD Vacuntainer 25x8mm descartáveis, tubos de coleta a vácuo Vacuntainer siliconizado sem anticoagulante e adaptador de agulhas BD Vacutainer.

\section{Análise do $\mathbf{M g}^{2+}$ plasmático}

A análise do $\mathrm{Mg}^{2+}$ foi realizada no analisador semi-automático BTS-310 Plus (BioSystems, Espanha), utilizando o Kit para magnésio Invitro (Brasil), utilizando o método azul de xilidina, no qual os íons magnésio produzem um complexo corado, em meio alcalino, frente ao corante de Mann e Yoe. No qual a intensidade da cor formada é proporcional à concentração de Magnésio, sendo a leitura realizada a $505 \mathrm{~nm}$.

\section{Análise estatística}

Os tratamentos dos dados foram realizados através da estatística descritiva e utilizando o teste $T$ de student para variáveis dependentes, através do pareamento dos dados (oriundos de um procedimento tipo antes-e-depois], com auxilio do programa SPSS (Statistical Packge for Social Sciences para Windows ${ }^{\circledR}$ ] versão 11.0 (SPSS Inc., Chicago, IL, USA, 2001). Foi adotado $\mathrm{p} \leq$ 0,05 como nível mínimo de significância. Segundo Thomas; Nelson (2002) este teste T de dados pareados, serve para avaliar a significância das diferenças das médias e de dois conjuntos de escores relacionado, tal como quando os sujeitos são medidos em duas ocasiões. 


\section{RESULTADOS}

Na tabela 1 são apresentados os tempos de natação, ciclismo, corrida e o tempo total da prova.

Os valores da concentração plasmática média de $\mathrm{Mg}^{2+}$ pré-competição no grupo experimental foi de 2,06 $\pm 0,241 \mathrm{mg} \mathrm{dL}-1$ sendo que os valores mínimos e máximos foram de $1,80 \mathrm{mg} \mathrm{dL}^{-1}$ e 2,40 $\mathrm{mg} \mathrm{dL}^{-1}$, respectivamente e no grupo controle a concentração média de $\mathrm{Mg}^{2+}$ foi de 1,98 $\pm 0,482 \mathrm{mg} \mathrm{dL}^{-1}$ onde os valores mínimo e máximo foram de $1,40 \mathrm{mg} \mathrm{dL}^{-1}$ e $2,50 \mathrm{mg} \mathrm{dL}^{-1}$ respectivamente (gráfico 1 ).

No grupo experimental, após a competição, a concentração plasmática média de $\mathrm{Mg}^{2+}$ foi de 1,32士 $\pm 0,084 \mathrm{mg} \mathrm{dL}^{-1}$ apresentado os valores mínimos e máximos de 1,20 $\mathrm{mg} \mathrm{dL}^{-1}$ e 1,40 $\mathrm{mg} \mathrm{dL}^{-1}$ respectivamente e no grupo controle

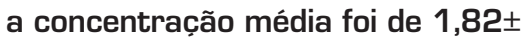
$\pm 0,178 \mathrm{mg} \mathrm{dL}^{-1}$ e os valores mínimos e máximos encontrados foram de 1,62 $\mathrm{mg} \mathrm{dL}^{-1}$ e 2,11 $\mathrm{mg} \mathrm{dL}^{-1}$ respectivamente (gráfico 1).

Quando comparado os valores da concentração $\mathrm{Mg}^{2+}$ no período antes da competição os valores do grupo controle e experimental não apresentaram diferença significativa $(\mathrm{P}=0,788)$. Porém, quando comparado os valores após a competição entre o grupo controle e o grupo experimental apresentou uma diferença significativa na concentração plasmática de $\mathbf{M g}^{2+}$, apresentando um valor de $\mathrm{P}=0,005$.

Os valores da concentração plasmática de $\mathrm{Mg}^{2+}$ no grupo experimental avaliados antes e após a competição de triathlon também foram avaliados e apresentou uma diferença significativa $(P=0,005)$, apresentando uma redução média de $35,23 \%$. Quando comparado os valores do grupo experimental após a

\begin{tabular}{lcccc}
\hline & Mínimo & Máximo & Média & Desvio-Padrão \\
\hline Natação & $0: 19: 31$ & $0: 26: 05$ & $0: 22: 14$ & $0: 02: 22$ \\
Ciclismo & $2: 42: 22$ & $2: 59: 30$ & $2: 51: 56$ & $0: 06: 23$ \\
Corrida & $1: 21: 28$ & $2: 22: 55$ & $1: 46: 20$ & $0: 23: 44$ \\
Tempo Total & $4: 23: 21$ & $5: 44: 07$ & $5: 00: 32$ & $0: 30: 48$ \\
\hline
\end{tabular}

TABELA1

Tempo de prova. competição com o grupo controle antes da competição apresentou também uma diferença significativa $(P=0,035)$. Não houve diferença significativa nos valores da concentração plasmática do grupo controle antes e após a competição $(P=$ $=0,399$ ), apresentando uma redução média de $4,48 \%$.

\section{DISCUSSÃO}

A duração, a intensidade e as condições ambientais extremas, encontradas nos eventos de ultra- -endurance combinam-se na geração de diferentes estresses fisiológicos diferentemente dos encontrados nos exercícios de endurance (Peters, 2003), podendo estes gerar um déficit de $\mathrm{Mg}^{2+}$ por vários mecanismos (Rayssiguier; Guezennec; Durlach, 1990).

A capacidade do organismo de sustentar uma atividade física extenuante ou prolongada pode ser reduzida, pela alta taxa de perda de eletrólitos pelo suor, no qual há perda significativa de magnésio (Riche, 1989; Brotherhood, 1984; Randerath; König; Jansen, 2004],

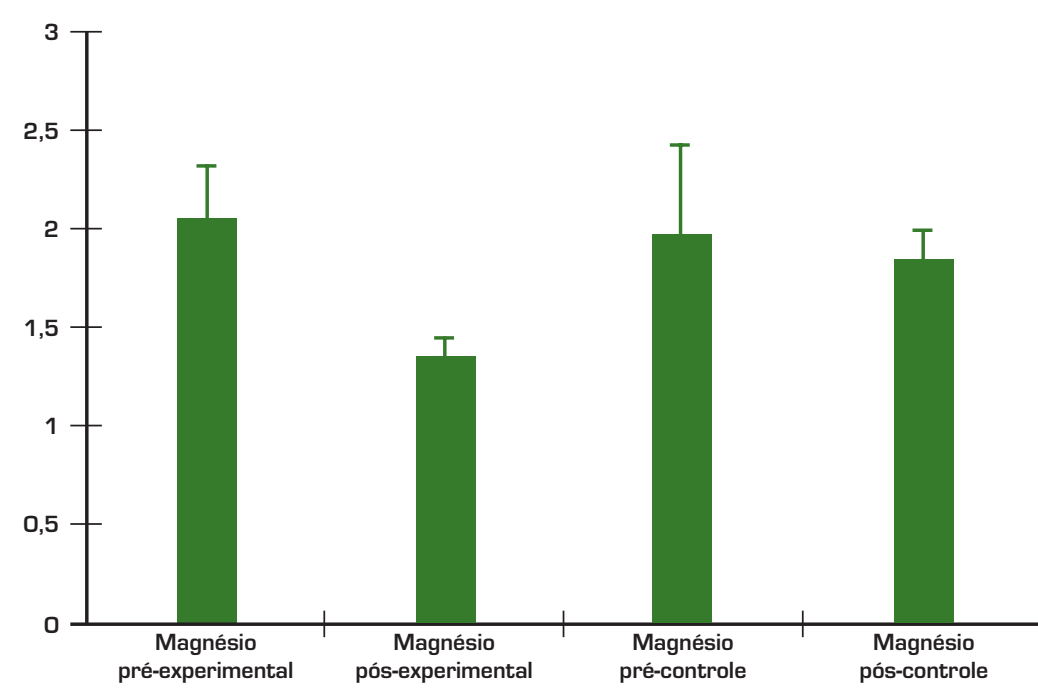

FIGURA1

Concentração $\mathrm{Mg}^{2+}$ plasmático expressos em $\mathrm{mg} \mathrm{dL}^{-1}$. 
podendo este ter uma concentração média de $0.8 \mathrm{mmol} \mathrm{L}^{-1}$ (variando 0.2-1.5 mmol L-1) (Sawka; Montain, 2000), causada pelo estresse induzido pelo exercício, por uma dieta desbalanceada e uma reposição eletrolítica inadequada (Riche, 1989). Experimentos em modelo animal têm demonstrado que a deficiência de $\mathrm{Mg}^{2+}$ reduz o desempenho, sendo relacionado à redução da geração de energia pelo metabolismo (Rayssiguier; Guezennec; Durlach, 1990). A redução da concentração de $\mathrm{Mg}^{2+}$ pode estar associada a um aumento na concentração plasmática de ácidos graxos causada pela lipólise (Rayssiguier; Guezennec; Durlach, 1990), no qual há um deslocamento do $\mathrm{Mg}^{2+}$ plasmático para dentro dos adipócitos (Warburton et al 2002), durante os eventos de longa duração, podendo estas alterações gerar espasmos musculares [Randerath; König; Jansen, 2004; Rayssiguier; Guezennec; Durlach, 1990), por não conseguir bloquear o efeito estimulante do cálcio nos músculos (Willians, 2002), gerando a perda da competitividade do atleta.

No estudo realizado por Costill; Cote; Fink (1976) foram analisados oito voluntários, pré e pós-exercício, sendo submetidos a diversos graus de desidratação, causados pela atividade física realizada numa bicicleta ergométrica com ambiente controlado, a qual se localizava numa sala com temperatura ambiente de $39,5^{\circ} \mathrm{C}$ com umidade relativa do ar em $25 \%$. Os voluntários se exercitaram até obter uma redução ponderal de $2 \%$, sendo este processo repetido até apresentarem os quadros de 4 e $6 \%$ de redução ponderal. Foram coletadas amostras de sangue a cada estágio de desidratação para análise da concentração plasmática de eletrólitos.
Quando comparados os valores antes e após a redução ponderal de $2 \%$, a concentração plasmática do $\mathrm{Mg}^{2+}$ apresentou uma redução significativa $(P<0.05)$. Estes valores se mantiveram estáveis nos quadros de 4 e $6 \%$ de redução ponderal, sendo que esses achados corroboram este estudo nos quais os valores da concentração plasmática $\mathrm{Mg}^{2+}$ apresentaram uma redução significativa quando comparado aos valores pré e pós-competição de triathlon. Quando comparados aos valores do grupo controle pré e pós-competição, apresentaram também uma redução significativa na concentração plasmática.

No estudo realizado por Schwellnus et al. (2004), no qual foram analisados 43 corredores de ultramaratona antes e após a corrida Two Oceans, com $56 \mathrm{~km}$ de distância, realizada anualmente na África do Sul. Os atletas que foram divididos em dois grupos: os que sofreram câimbras durante algum trecho da prova e atletas que não apresentaram câimbras (controle). Não foi encontrada uma diferença significativa entre a concentração sérica do $\mathrm{Mg}^{2+}$ pré-competição em ambos os grupos, sendo estes resultados semelhantes ao encontrados no presente estudo e ao termino da corrida a concentração do $\mathbf{M g}^{2+}$ apresentou-se significativamente elevada no grupo que sofreu câimbras do que o controle diferentemente dos achados desse estudo, onde os voluntários apresentaram uma redução significativa da concentração plasmática.

No estudo de Astier et al. (1996), foi demonstrado que a deficiência de $\mathrm{Mg}^{2+}$ no músculo esquelético gera alterações físico-químicas e bioquímicas na membrana do reticulo sarcoplasmático, sendo este o maior sistema envolvido na ho- meostasia do cálcio no músculo esquelético (Astier et al., 1996). Demonstrando que a deficiência de $\mathrm{Mg}^{2+}$ afeta as estruturas e as propriedades funcionais da membrana do reticulo sarcoplasmático do músculo esquelético. Zimmermann (2003) sugere que a suplementação de $\mathrm{Mg}^{2+}$ não afeta a performance quando os níveis séricos se encontram dentro dos valores de normalidade, podendo ter um ganho de performance quando se tem nível marginal de $\mathbf{M g}^{2+}$.

Segundo Liu; Borowski; Rose (1983, apud Lukaski, 2004), a deficiência de $\mathrm{Mg}^{2+}$ reduziu a performance dos jogadores de tennis, sendo esta associada a espasmos musculares, diferentemente dos achados de Sulzer; Schwellnus; Noakes (2005) onde foram estudados 20 triathletas após uma competição de triathlon na distância ironman [1,8 km de natação, 180 km de corrida e 42 de corrida). Sendo estes 20 divididos em dois grupos: um grupo com 11 atletas que sofreram câimbra durante a prova e outro grupo com 9 atletas que não sofreram câimbras. Quando comparados os valores da concentração plasmática de $\mathrm{Mg}^{2+}$ não houve diferença significativa entre os dois grupos. Segundo O'Toole; Douglas (1995, apud Jeukendrup et al. 2005) a hipomagnesemia pode ser um problema nas competições de triathlon de longa duração.

No estudo realizado por Lijnen et al. (1988), foram analisados 23 maratonistas antes, imediatamente ao término da maratona e após 12 horas de descanso, com o tempo médio de duração da prova de $3 \mathrm{~h}$ 41 min. Quando comparado os valores de $\mathrm{Mg}^{2+}$ no plasma, verificou-se uma redução significativa $(P<$ $<0.05$ ), apresentando uma redução de $8 \%$ na concentração do $\mathrm{Mg}^{2+}$ no 
plasma sangüíneo. Quando comparado à concentração de $\mathbf{M g}^{2+}$ dentro dos eritrócitos antes e ao término da maratona, estes valores apresentaram uma redução de $5 \%$, sendo esta significativa quando comparado aos seus valores antes da corrida. Os valores após 12 horas do término da corrida apresentaram-se dentro da faixa de normalidade. Esses achados corroboram o presente estudo no qual verificou após o término da prova de triathlon que houve uma redução significativa na concentração do $\mathrm{Mg}^{2+}$ plasmático, indicando que o exercício de ultra-endurance aumenta significativamente a eliminação de $\mathrm{Mg}^{2+}$, possivelmente relacionado ao aumento da sudorese, o aumento da taxa metabólica e o estresse muscular sofrido durante o exercício de ultra-endurance.

No estudo de Welsh et al. (1999), não se verificou diferença na concentração plasmática de $\mathrm{Mg}^{2+}$, em nove voluntários após uma prova de triathlon de longa distância, sendo este resultado diferente do presente estudo.

No estudo realizado por Golf; Bender; Grüttner (1998) no qual foram analisados 25 triathletas, após quatro semanas com suplementação ou placebo ao término do período foram submetidos a uma prova simulada de triathlon, no qual foram coletadas amostras de sangue antes e após o término de cada modalidade. A concentração plasmática de magnésio apresentou-se inalterada nos dois grupos, porém a concentração de magnésio nos eritrócitos aumentou no grupo suplementado e no grupo controle apresentou uma redução, sendo que em ambos os grupos apresentaram alterações significantes ao término do estudo. Os achados desse estudo corroboram os achados de Golf et al. (1984) no qual ele postula que o aumento na duração do exercício acarreta uma troca entre as reservas de magnésio localizadas nos eritrócitos com o sangue e desta com os músculos em atividade, para manter a concentração do magnésio plasmático dentro dos valores fisiológicos.

Segundo Evans; Haliwell, (2001) e Astier et al. (1996) a deficiência de $\mathrm{Mg}^{2+}$ pode aumentar a susceptibilidade das células e tecidos a peroxidação lipídica. Sendo que aumento da geração de radicais livres (RL) também está associado com a lesão muscular com um significante aumento na concentração do cálcio (Astier et al., 1996).

\section{CONCLUSÃO}

Os resultados obtidos no presente estudo corroboram com a literatura disponível, no qual a alteração da concentração dos íons $\mathbf{M g}^{2+}$ pode estar relacionada um aumento na concentração plasmática de ácidos graxos causada pela lipólise, pela perda excessiva de íons (Rayssiguier; Guezennec; Durlach, 1990), tanto pelo suor como pelo aumento no estresse muscular (Speich et al., 2001), durante os eventos de longa duração, podendo estas alterações gerar espasmos musculares (Randerath; König; Jansen, 2004; Rayssiguier; Guezennec; Durlach, 1990), por não conseguir bloquear o efeito estimulante do cálcio nos músculos (Willians, 2002), gerando a perda da competitividade do atleta.

Para um melhor entendimento dos processos fisiológicos envolvidos é de suma importância a realização de experimentos que analisem a concentração de íons no suor em ambiente laboratorial para que se tenha noção de quanto e a perda desses íons no suor. Além disto, a análise de marcadores relacionados ao metabolismo lipídico, também se faz necessária, pois o aumento da taxa de metabolismo deste nutriente esta relacionada com a redução da concentração do $\mathbf{M g}^{2+}$ plasmático, sendo que os lipídios são de uma importância ímpar nas provas de longa duração no qual é a principal fonte energética do organismo.

\section{CORRESPONDÊNCIA}

Marcos Bürger-Mendonça

Rua Paulo Barbosa 14/1102

Petrópolis, Rj, Brasil

Cep: 25620-100

\section{REFERÊNCIAS}

1. Astier C, Rock E, Lab C, Gueux E, Mazur A, Rayssiguier Y. Functional alterations in sarcoplasmic reticulum membranes of magnesium-deficient rat skeletal muscle as consequences of free radical-mediated process. Free Radic Biol Med. v. 20, n. 5, pp. 667-74, 1996.

2. Bohl $\mathrm{CH}$, Volpe SL. Magnesium and exercise. Crit Rev Food Sci Nutr. v.46, n.6, pp.533-563, 2002.

3. Brotherhood JR. Nutrition and sports performance. Sports Med. v. 1, n. 5, pp. 350-389, 1984. (Resumo).

4. Costill DL, Cote R, Fink W. Muscle water and electrolytes following varied levels of dehydration in man. J Appl Physiol. v. 40, n. 1, pp. 6-11, 1976. 
5. Deuster PA, Dole E, Kyle SB, Anderson RA, Schoomaker EB. Magnesium homeostasis during high-intensity anaerobic exercise in men. J Appl Physiol. v. 62, n. 2, pp. 545-550, 1987.

6. Evans P, Halliwell B. Micronutrients: oxidant/antioxidant status. Br J Nutr. v. 85, n. s2, p. 5 67-74, p. 2001.

7. Gay R, Meydan SM. The effects of vitamin $E$, vitamin B6, and vitamin B12 on immune function. Nutr Clin Care. v. 1, n. 4, pp. 188-198, 2001.

8. Golf SW, Bender S, Grüttner J. On the Significance of Magnesium in Extreme Physical Stress. Cardiovasc Drugs Ther. v. 12, pp. 197. -202, 1998.

9. Golf SW, Happel O, Graef V, Seim KE. Plasma aldosterone, cortisol and electrolyte concentrations in physical exercise after magnesium supplementation. J Clin Chem Clin Biochem. v. 22, n. 11, pp. 717-21, 1984 (Resumo).

10. Jeukendrup $A E$, Jentjens RL, Moseley L. Nutritional considerations in triathlon. Sports Med. v. 5, n. 2, pp. 163-181, 2005.

11. Lijnen P, Hespel P, Fagard R, Lysens R, Vanden Eynde E, Amery A. Erythrocyte, plasma and urinary magnesium in men before and after a marathon. Eur J Appl Physiol Occup Physiol. v. 58, n. 3, pp. 252 -256, 1988.

12. Lukaski HC. Vitamin and mineral status: effects on physical perfor- mance. Nutrition. v. 20, n. 7-8, pp. 632-644, 2004.

13. Peters EM. Nutritional aspects in ultra-endurance exercise. Curr Opin Clin Nutr Metab Care. v. 6, n. 4, pp. 427-434, 2003.

14. Randerath O, König DP, Jansen T. Magnesiumsubstitution im breiten-und leistungssport. [Magnesium substitution in professional and popular sport] Biologische Medizin. v. 33, n. 4, pp. 178-181, 2004. (Resumo).

15. Rayssiguier Y, Guezennec CY, Durlach J. New experimental and clinical data on the relationship between magnesium and sport. Magnes Res. v. 3, n. 2, pp. 93-102, 1990. 16. Recomendações da Sociedade Brasileira de Patologia Clínica/ /ML para Coleta de Sangue Venoso, $1^{\text {a }}$ Ed./elaborado pelo Comitê de Coleta de Sangue da SBPC/ML e BD Diagnostics - Preanalytical Systems. São Paulo, 2005.

17. Riche $D$. Les déficits en magnésium et le sport. Science \& Sports. v. 4, n. 1, pp. 41-52, 1989. (Resumo).

18. Sawka MN, Montain SJ. Fluid and electrolyte supplementation for exercise heat stress. Am J Clin Nutr. v. 72, n, 2 Suppl, p. 5645-572s, 2000.

19. Schwellnus MP, Nicol J, Laubscher R, Noakes TD. Serum electrolyte concentrations and hydration status are not associated with exercise associated muscle cramping (EAMC) in distance runners. $\mathrm{Br} \mathrm{J}$ Sports Med. v. 38, n. 4, pp. 488-492, 2004.
20. Speich M, Pineau A, Ballereau F. Minerals, trace elements and related biological variables in athletes and during physical activity. Clin Chim Acta. v. 312, n.1-2, pp. 1-11, 2001.

21. SPSS Statistical Packge for Social Sciences for Windows ${ }^{\circledR}$ versão 11.0 SPSS Inc., Chicago, IL, USA, 2001.

22. Sulzer NU, Schwellnus MP, Noakes TD. Serum electrolytes in Ironman triathletes with exercise-associated muscle cramping. Med Sci Sports Exerc. v. 37, n. 7, pp. 1081-1085, 2005.

23. Thomas JR, Nelson JK. Métodos de pesquisa em atividade física. Tradução Ricardo D. S. Petersen. Porto Alegre: ARTMED, 2002.

24. Warburton DE, Welsh RC, Haykowsky MJ, Taylor DA, Humen DP. Biochemical changes as a result of prolonged strenuous exercise. $\mathrm{Br} J$ Sports Med. v. 36, n. 4, pp. 301-303, 2002.

25. Welsh RC, Warburton DER, Haykowsky MJ, Taylor DA, Humen Dennis P. Hematological and biochemical response to a half Ironman triathlon. Med Sci Sports Exerc. v. 31, n. 5, p. $S$ 140, 1999.

26. Williams MH. Nutrição para Saúde, Condicionamento Físico e Desempenho Esportivo.Tradução Sonia Bidutte, São Paulo: Manole, 2002.

27. Wolf FI, Cittadini A. Chemistry and biochemistry of magnesium. 